\title{
STIMOLARE LA PARTECIPAZIONE ALLA CONVERSAZIONE IN CLASSI CON LIVELLO ETEROGENEO DI SCOLARIZZAZIONE. LE PRATICHE DEL TEATRO SOCIALE PER SVILUPPARE SCOPI COMUNICATIVI REALI: \\ L'ESPRESSIONE DI SÉ E L'INTEGRAZIONE
}

\author{
Claudia Canuto ${ }^{1}$
}

\section{INTRODUZIONE}

L'obiettivo della presente ricerca è quello di indagare pratiche e prospettive che permettano agli insegnanti di lingua di stimolare la conversazione nelle classi ad abilità differenziate (Caon, 2016). Le interazioni nelle classi di lingua sono caratterizzate da una forte asimmetria e assenza di spontaneità a causa di una distribuzione "diseguale" dei diritti comunicativi fra gli interattanti: l'insegnante detiene la maggior parte dello spazio di parola, gestisce i turni degli apprendenti e seleziona i temi della conversazione (Fele, 2007; Fele, 2003; Nuzzo, Grassi, 2016; Orletti, 2000). Tale organizzazione gerarchica non favorisce l'apprendimento della seconda lingua che necessita di scambi comunicativi e possibilità dialogiche. La produzione in L2 è infatti cruciale ai fini dello sviluppo della competenza d'uso in quanto sottopone a verifica le ipotesi sul funzionamento della lingua da apprendere; grazie alle reazioni degli interlocutori (correzioni, richieste di chiarimento, manifestazioni di mancata comprensione, riformulazioni, eterocorrezioni) automatizza l'uso delle strutture apprese, rendendo più fluente l'eloquio; stimola l'apprendente a passare da un'elaborazione semantica a una morfosintattica; il discente, nel tentativo di essere compreso, si sforza di produrre un enunciato grammaticalmente strutturato (Grassi, 2017; Nuzzo, Grassi, 2016; Rastelli, 2010).

Nella classe di L2, l'adozione di determinate tecniche didattiche (giochi di ruolo, conversazioni a coppie, dibattiti, progetti, pratiche teatrali, giochi didattici) può scardinare l'organizzazione gerarchica della conversazione: l'insegnante incentiva la presa di parola libera, promuove l'interazione spontanea, coinvolge gli studenti nella scelta dei contenuti e lascia loro maggiore spazio di parola (Diadori, Palermo, Troncarelli, 2015). In particolare, la classe di italiano L2 per persone migranti, avendo come fine ultimo l'integrazione civica e linguistica dei propri discenti, che spesso vivono in condizioni di isolamento sociale, necessita di riprodurre al suo interno uno spazio di interazione e confronto (Minuz, 2014).

L'interesse della presente ricerca, condotta nel 2018 presso la città di Torino ${ }^{2}$, consiste dunque nell'osservazione di due laboratori di teatro sociale rivolti a persone migranti, richiedenti asilo e rifugiati politici, al fine di mettere in luce la diversa relazione fra gli attori dello scambio comunicativo. L'uso della drammatizzazione all'interno di un percorso

\footnotetext{
${ }^{1}$ Università degli Studi di Torino, co-coordinatrice didattica del progetto "Italiano L2 a scuola".

2 Percorso di ricerca per la stesura della tesi di laurea magistrale presso l’Università di Torino, sotto la guida della professoressa Cecilia Andorno. Si ringraziano Cecilia Andorno e Paolo della Putta per la revisione scientifica e le possibilità di confronto; mancanze ed errori sono responsabilità dell'autrice.
} 
glottodidattico propone un ribaltamento delle dinamiche tipiche dell'interazione in classe, in quanto $i$ discenti vengono coinvolti in scopi comunicativi veri: l'insegnante si pone come elemento di avvio delle attività e come facilitatore, i discenti interagiscono e cooperano nel corso di pratiche il cui obiettivo è l'espressione di sé (Comodi, 1999; Fonio, 2013; Galli, 2018; Magnani, 2002; Piazzoli, 2009). Per rilevare i dati e per verificare l'utilità delle attività nello sviluppo delle dinamiche di interazione sono stati usati gli strumenti dell'Analisi della Conversazione.

Nel primo paragrafo si illustrano le premesse teoriche che sostengono la ricerca: le dinamiche dell'interazione nella classe di lingua e le caratteristiche delle classi eterogenee di italiano L2 per persone migranti. Nel secondo paragrafo si offre un breve inquadramento sulla glottodidattica teatrale e la definizione di teatro sociale. Nel terzo paragrafo si descrive la ricerca e il contesto in cui si è svolta, nel quarto si analizzano i risultati e, infine, nel quinto paragrafo si discutono le conclusioni.

\section{PREMESSE TEORICHE}

\subsection{La partecipazione nella classe di lingua: le dinamiche dell'interazione in classe}

La conversazione in classe viene definita da Orletti come interazione diseguale o asimmetrica in quanto i partecipanti non hanno pari diritti comunicativi. Lo scambio comunicativo avviene fra un gruppo (la classe) e un singolo (l'insegnante), quest'ultimo assume un ruolo di guida e regia, esercitando il potere di controllo e di dominanza sullo scambio comunicativo: gestisce i turni, selezionando e scegliendo gli interlocutori (dominanza interazionale); usa le domande per veicolare i contenuti e assegnare i turni (dominanza strategica); stabilisce la durata dei turni, interrompendo gli interlocutori e occupando la maggior parte dello spazio di parola (dominanza quantitativa); seleziona i temi e i contenuti dell'interazione (dominanza semantica) (Fele, 2003; Orletti, 2000).

Per quel che riguarda la gestione dei turni da parte dell'insegnante di lingua è necessario soffermarsi su tre aspetti: la struttura triadica dell'interazione, l'assegnazione dei turni e la loro durata. La struttura triadica è una delle caratteristiche principali della conversazione nella classe di lingua ed è così organizzata:

- primo turno, rigorosamente iniziato da un insegnante, che formula una domanda a un discente (o alla classe intera);

- secondo turno, in cui lo studente risponde;

- terzo turno, in cui l'insegnante valuta la risposta del discente e inizia la sequenza successiva, precludendo possibilità di reazione allo studente.

Tabella 1. Turni e tipi di conversazione

\begin{tabular}{|l|l|}
\hline \multicolumn{1}{|c|}{ Conversazione ordinaria } & \multicolumn{1}{c|}{ Conversazione in classe } \\
\hline Interlocutore 1: che giorno è oggi? & Insegnante: Henry, che giorno è oggi? \\
Interlocutore 2: venerdì & Studente: venerdì \\
Interlocutore 1: grazie & Insegnante: bravo! \\
\hline
\end{tabular}

Nelle conversazioni ordinarie, il terzo turno può essere usato da chi ha avviato la domanda per commentare, esprimere un parere o per dare segnali di ricezione di ascolto 
("grazie"). Nell'ambito scolastico il terzo turno viene invece usato a fini valutativi ("bravo!"); inoltre chi occupa il terzo turno è anche la stessa persona che darà l'avvio alla sequenza successiva. Infatti, se le conversazioni ordinarie hanno una struttura binaria, ossia c'è un alternarsi fra i due interlocutori (interlocutore 1 - interlocutore 2 ; interlocutore 1- interlocutore 2), i quali possono scegliere se commentare, accettare o rifiutare il turno, nella conversazione scolastica la struttura è ternaria (insegnante-studente-insegnante).

La tripletta didattica è stata considerata artificiosa, in quanto funzionale unicamente alla scuola, ed è stata criticata in quanto potrebbe abbassare il coinvolgimento dei discenti a intervenire nella conversazione. In questo modo i contributi degli studenti risultano essere talmente ridotti da non permettere un avanzamento nello sviluppo dell'interlingua (Nuzzo, Grassi, 2016; Pallotti, 2012). Inoltre, come sottolinea Pallotti (2012: 273-277) tale struttura è artificiosa, in quanto usata quasi esclusivamente nell'interazione scolastica, e non riproduce ciò che accade realmente nelle conversazioni spontanee.

Nonostante le critiche a questa forma scolastica di interazione, va notato che il terzo turno assume un ruolo importante per gli apprendenti, i quali si sentono rassicurati nel ricevere conferme sulla propria produzione da parte del parlante esperto (Chini, 2018; Fele, 2003). Tale feedback viene distinto da Chini (2018: 116) in:

a) mosse di approvazione, accettazione, conferma di quanto detto dal discente;

b) interventi e commenti metalinguistici sulla produzione dell'apprendente;

c) richieste di chiarimento o conferma espresse con vari tipi di domande.

Infatti, non tanto nel terzo turno, piuttosto nel primo possiamo individuare la principale causa di assenza di spontaneità dell'interazione in classe (Nuzzo, Grassi, 2016). Per una conversazione più autentica e comunicativa è necessario che l'insegnante non ponga domande esclusivamente mirate a verificare che l'interlocutore conosca la risposta o sappia rispondere con una forma corretta: «è la differenza fra "Chi ha scoperto l'America?" e "Chi reputi sia stato il più grande esploratore di tutti i tempi?"» (ivi: 128). Le domande di esibizione sono quasi assenti nelle conversazioni spontanee, mentre rappresentano dai tre quarti fino addirittura ai nove decimi delle domande che gli insegnanti pongono ai loro discenti (Pallotti, 2012). Queste, oltre a non riflettere ciò che avviene realmente in uno scambio comunicativo, tendono a produrre risposte brevi e poco complesse anche dal punto di vista linguistico e non stimolano il pensiero dei discenti (Minardi, 2020).

Un altro fattore che caratterizza la disparità comunicativa della classe di lingua riguarda la selezione dei partecipanti alla conversazione: è quasi sempre l'insegnante a scegliere gli interlocutori, rivolgendo una domanda all'intera classe e, in caso di mancata risposta, assegnando il turno a un determinato discente (Pallotti, 2012). È raro che siano gli studenti a formulare domande o ad autoselezionarsi per condividere una riflessione. Sono scarse anche le possibilità dei discenti di selezionare un interlocutore che non sia il docente. Inoltre, quando avviene l'autoselezione da parte di uno studente, questa è quasi sempre per alzata di mano (Fele, 2003). Queste sequenze autoiniziate dai discenti hanno strutture ricorrenti: richiesta di autorizzazione e conseguente accettazione o rifiuto da parte dell'insegnante (Orletti, 2000).

Infine, l'ultimo aspetto che evidenzia il potere del docente nella gestione dei turni riguarda la dominanza quantitativa che caratterizza lo spazio di parola del docente: la media che emerge da diversi studi è che «l'insegnante parla circa il $70 \%$ del tempo, contro il 30\% lasciato agli allievi: se si considera che questi sono $20-30$ per classe, si arriva alla conclusione che ognuno di loro ha la possibilità di parlare poco più di 30 secondi all'ora» (Pallotti, 2012: 84). Un'altra spiegazione alla quantità di spazio conversazionale occupata dagli insegnanti è che questi non concedono agli apprendenti il tempo sufficiente per 
rispondere alle domande. Tale forma di impazienza viene giustificata da alcuni docenti, intervistati in uno studio di Tsui (1996), come risposta al timore di perdere il controllo della classe o come forma di imbarazzo in presenza di silenzio. Le conseguenze del potere del docente sulla conversazione nella classe di lingua porta quindi ad interazioni statiche e passive.

\subsection{La classe di italiano L2 per persone migranti}

L'eterogeneità è una caratteristica che contraddistingue le classi di italiano L2 per persone migranti. Questa riguarda l'età dei partecipanti, la provenienza, le lingue conosciute, i contesti culturali di origine, i documenti, il progetto migratorio, la motivazione ad apprendere, il livello di scolarizzazione e la condizione sia sociale sia di vita nel Paese di arrivo (Trulli, 2014). Ognuno di questi fattori ha un ruolo decisivo nell'apprendimento della seconda lingua e condiziona l'andamento e la struttura delle lezioni. Infatti, le classi di italiano per persone migranti rientrano nella definizione di classi ad abilità miste, ossia classi con studenti differenti tra loro per «livello linguistico, background culturale, stili di apprendimento, attitudine verso la lingua, madrelingua, intelligenza, conoscenza del mondo, esperienza di apprendimento, conoscenza di altre lingue, età o maturità, genere, personalità, fiducia in sé stessi, motivazione, interesse, indipendenza, autodisciplina e livello di educazione» (Caon, 2016: 138).

In questa sede ci soffermeremo brevemente su una di queste categorie: i diversi livelli di scolarizzazione. In una classe di italiano L2 per persone migranti, soprattutto nei contesti di volontariato, si possono facilmente trovare apprendenti che non hanno frequentato la scuola nel paese di origine, che hanno conseguito la licenza media, un diploma superiore o la laurea (Trulli, 2014). Le ricerche sulla pratica didattica e sull'acquisizione portate avanti dal gruppo LESLLA ${ }^{3}$ mettono in luce che si crea un divario fra gli apprendenti alfabetizzati e quelli scarsamente o non alfabetizzati. Tale divario si riverbera nei:

a) tempi di apprendimento della lingua, che sono radicalmente più lunghi in soggetti LESLLA;

b) risultati del processo di apprendimento che vedono precoci e radicali fossilizzazioni (cioè dei veri e propri definitivi inceppamenti del processo di acquisizione della lingua) nei parlanti LESLLA (Amoruso, D’Agostino 2016: 24).

Come osservano Amoruso e D'Agostino (2016) in merito agli studenti iscritti ai corsi dell'Università per Stranieri di Palermo, i differenti livelli di scolarizzazione comportano la costruzione di classi a diverse andature: negli studenti con nessun anno di scolarità si registra il mancato riconoscimento di categorie concettuali (nome, aggettivo, verbo) e la possibilità di individuare i confini fra le parole; negli apprendenti con pochi anni di scolarizzazione si presenta invece una difficoltà notevole nella comprensione $\mathrm{e}$ produzione di testi scritti, nel reperire e adottare strategie e nel comprendere le consegne, oltre che una ridotta capacità di riflessione metalinguistica. Invece, gli studenti che hanno alle spalle un solido percorso scolastico possono attivare le conoscenze pregresse e le strategie di apprendimento per guidare l'acquisizione della nuova lingua (Cognini, 2019).

\footnotetext{
${ }^{3}$ L'acronimo Leslla (Literacy Education and Second Language Learning for Adults) fa riferimento a un gruppo di ricerca internazionale che si occupa di studiare metodi per l'acquisizione linguistica e l'alfabetizzazione di apprendenti migranti con scarsa o assente esperienza di scolarizzazione nel paese d'origine (https://www.leslla.org/).
} 
Come sottolineano De Meo e Maffia (2021: 120), il basso livello di scolarizzazione nella lingua materna o nella lingua di istruzione può fra l'altro accentuare la condizione di vulnerabilità degli apprendenti:

in un contesto abitativo tutt'altro che semplice, quale quello delle strutture di accoglienza, l'analfabetismo diventa, quindi, causa di un ulteriore trauma, definito "shock da documento" (Adami, 2009): una sensazione di disorientamento semiotico e, quindi, di inadeguatezza, che può sfociare nei casi più gravi in autocensura, con il rischio di essere sottoposti facilmente a manipolazione da parte di chi padroneggia gli strumenti della lettura e della scrittura.

Un altro fattore da tenere in considerazione quando si osserva il diverso livello di scolarizzazione degli apprendenti migranti è l'eterogeneità della qualità dell'istruzione: è molto frequente che chi ha frequentato la scuola coranica si dichiari scolarizzato in sede di iscrizione alla scuola. La scuola coranica si concentra prevalentemente «sulle abilità di ascolto, produzione orale e memorizzazione di testi orali, esercitate attraverso la ripetizione del Corano» (De Meo, 2018: 57). In questi casi la frequenza scolastica, anche per diversi anni, non è certo che porti all'alfabetizzazione: nelle scuole coraniche si dà per lo più spazio alla memorizzazione del testo sacro, rispetto alla sua lettura (D'Agostino, Lo Maglio, 2018: 26). Secondo D'Agostino e Lo Maglio (2018), nelle scuole coraniche scolarizzazione e alfabetizzazione non si equivalgono: il processo di memorizzazione del testo sacro non porta a una reale decifrazione del testo ma solo al suo riconoscimento. A fronte di queste riflessioni D'Agostino e Lo Maglio (2018: 26) mettono in luce quanto l'alfabetizzazione ricavata da test e dall'autodichiarazione della propria scolarizzazione non siano realtà sovrapponibili.

Un secondo elemento che ci interessa mettere in luce in questa sede è la scarsa esposizione alla lingua seconda da parte degli apprendenti migranti: questo fattore si lega in particolare alla tipologia dei documenti posseduti, al progetto migratorio e alla condizione sociale e di vita (Amoruso, D’Agostino, Jaralla, 2015). Risultano essere poco esposte alla seconda lingua:

a) le donne che, per motivi familiari o culturali, vivono un contesto di isolamento sociale;

b) le persone che si sono ricongiunte ai propri familiari, che tendono a confrontarsi con la comunità di appartenenza o con la propria famiglia;

c) le persone ospitate nei progetti di accoglienza per richiesta di asilo politico, i quali hanno possibilità di socializzare prevalentemente con altri beneficiari (Amoruso, D’Agostino, Jaralla, 2015; Fiorucci, 2014; Trulli 2014).

Il terzo fattore che interessa questa ricerca riguarda la condizione di vulnerabilità degli apprendenti, che è prevalentemente caratterizzato da:

a) la mancata scelta di intraprendere un percorso migratorio, in quanto fuggiti da contesti di guerra e persecuzioni;

b) la frustrazione del lungo percorso di ottenimento delle procedure per il riconoscimento dello status di rifugiato politico;

c) la precarietà delle condizioni di vita.

In particolare, la precarietà è uno dei fattori che rende complessa l'iscrizione nelle scuole per la sua imprevedibilità a breve e lungo termine: alcuni apprendenti vengono infatti inseriti in strutture di accoglienza, altri decidono di lasciare l'Italia per raggiungere altri Paesi europei, altri ancora ottengono un lavoro e si trovano costretti ad abbandonare 
i percorsi formativi intrapresi. La precarietà di vita gioca quindi un ruolo fondamentale nella frequenza incostante delle lezioni: fra le cause di abbandono del pubblico femminile troviamo soprattutto la maternità, le incombenze domestiche e la necessità di badare ai figli piccoli (Trulli, 2014). Fra le altre difficoltà ricorrenti, relative all'abbandono dei corsi o alla frequenza incostante, Fiorucci e Trulli rilevano la scarsa disponibilità di tempo e la conseguente difficoltà a conciliare tempi da dedicare alla propria formazione con tempi di vita e di lavoro; l'assenza di una rete familiare che compensi la carenza dei servizi di cura; le difficoltà socio-culturali, come l'incapacità di fare emergere il bisogno di formazione; la necessità di inviare denaro ai familiari e le condizioni di lavoro spesso precarie, senza garanzie contrattuali e di bassa qualifica (Fiorucci, 2014; Trulli, 2014).

Minuz (2014: 109) sintetizza così il compito degli insegnanti di L2 per persone migranti: «facilitare lo sviluppo della competenza linguistico-comunicativa di donne e uomini adulti, che vivono, lavorano, agiscono socialmente in Italia, e imparano l'italiano come L2, in un contesto plurilingue». La classe di lingua per persone migranti deve tenere conto di diverse necessità: la lingua come strumento di integrazione; le diverse condizioni di vita; il differente interesse per i contesti di apprendimento, per gli ambiti e modi d'uso della lingua (Minuz, 2014). Inoltre, l'approccio usato in classe dovrebbe rispettare la ricchezza e la varietà d'uso dei repertori degli apprendenti: «questo non solo perché una didattica in prospettiva plurilingue meglio risponde alle caratteristiche dell'apprendente adulto e ai suoi reali comportamenti linguistici, ma anche e soprattutto perché il riconoscimento e il rispetto delle lingue degli altri sono un riconoscimento attivo della società multilingue e multiculturale che è oggi l'Italia» (ivi: 110). Gestire un corso di lingua che tenga conto di tutte queste necessità è un compito molto complesso, infatti, come osserva Della Putta (2021: 239) «gli insegnanti sviluppano sovente sentimenti di inadeguatezza e frustrazione verso i loro compiti, la cui oggettiva, intrinseca complessità è percepita come raramente sormontabile».

Una proposta di didattica che adotti alcune pratiche teatrali o che abbracci la pedagogia del teatro sociale, può lenire, almeno in parte, alcune delle difficoltà appena citate: le pratiche del teatro sociale, avendo come obiettivo l'espressione di sé, stimolano l'interazione coinvolgendo gli studenti in momenti di comunicazione vera. I discenti, essendo coinvolti in attività che hanno come obiettivo lo scambio, la costruzione del gruppo classe e l'espressione, sono protagonisti degli scambi comunicativi e hanno la possibilità di avere un ruolo determinante nella scelta dei contenuti delle lezioni, aiutando l'insegnante a mettere al centro della lezione $\mathrm{i}$ bisogni degli apprendenti e rispondendo al bisogno di autodeterminazione dei discenti adulti.

\section{IL TEATRO SOCIALE COME PRATICA PER STIMOLARE SCOPI COMUNICATIVI REALI}

\subsection{La glottodidattica teatrale}

A partire dagli anni Sessanta del secolo scorso, nell'ambito della glottodidattica, assumono maggior rilievo i limiti di un insegnamento basato unicamente sullo sviluppo della competenza linguistica, intesa come conoscenza del lessico e delle strutture grammaticali. Hymes introduce infatti il concetto di competenza comunicativa, che consiste nella «capacità di relazionarsi verbalmente e non verbalmente in modo efficace con individui che appartengono ad una cultura diversa dalla propria» (cit. in Ciliberti, 1994: 139). Nella seconda metà degli anni Settanta si sviluppa inoltre il concetto di "livello soglia", considerato come «il livello minimo di competenza linguistica utile per capire e 
farsi capire nella quotidianità» (Bosisio, Chini, 2014: 210). Viene quindi riconosciuta l'esigenza di raggiungere nella L2 un certo grado di competenza comunicativa che permetta alla persona straniera di lavorare, studiare e instaurare rapporti personali con i nativi. Negli anni successivi la ricerca si concentra sui fattori interni all'individuo che influenzano l'apprendimento: la motivazione, l'effetto delle emozioni sull'apprendimento, l'età. Si afferma così l'approccio umanistico, in cui viene ancor più valorizzato il ruolo dell'apprendente e soprattutto delle sue caratteristiche emotive. Nel programmare le lezioni diventa fondamentale prestare attenzione all'atmosfera della classe, alla costruzione di un ambiente rilassante, alla motivazione e al coinvolgimento degli studenti.

$\grave{E}$ in questo panorama che il teatro si è fatto avanti nella glottodidattica: «la glottodidattica teatrale ebbe origine in Gran Bretagna tra gli anni Cinquanta e Sessanta del secolo scorso quando, sull'onda della pedagogia umanistico-affettiva, se ne intravidero le indubbie potenzialità per stimolare la creatività e favorire la crescita» (Milici, 2019: 65). Le pratiche di drammatizzazione favoriscono innanzitutto la cooperazione fra gli apprendenti, in quanto questi dovranno interagire nel contesto sociale, risolvere problemi di gruppo e assumersi la responsabilità dei compiti che gli verranno assegnati (Magnani, 2002). Attività di questo tipo influiscono positivamente sulla motivazione del discente, a causa dello sviluppo dell'interazione sociale e dell'abbassamento del livello di ansia dei partecipanti. In particolare, quest'ultimo si riduce grazie al ridimensionamento della gerarchia fra insegnanti e discenti: l'insegnante assume un ruolo di regia e di facilitatore, lavorando soprattutto alla costruzione di un ambiente sereno, ludico e non competitivo (Galli, 2018; Piazzoli, 2009). Durante l'organizzazione di un laboratorio teatrale, il ruolo del docente si modifica: egli si pone come elemento di avvio alle attività e come figura di riferimento per le riflessioni linguistiche. Il docente scompare nell'ombra: è presente, ma lascia che si sviluppino liberamente i confronti e le discussioni fra pari (Comodi, 1999). L'uso del teatro a fini glottodidattici modifica, inoltre, la relazione fra i discenti: se nelle classi di conversazione troviamo gli studenti seduti, in file, rispettando i ritmi dettati dal docente, durante i laboratori di teatro le interazioni fra pari sono gestite quasi interamente dagli studenti stessi (Comodi, 1999; Milici, 2019; Piazzoli, 2009).

Coinvolgere gli apprendenti in pratiche teatrali permette una comparazione contrastiva tra i meccanismi della L1 e quelli della LS/L2: volume della voce, modulazione, velocità, ritmo, intonazione e segni non verbali; le nuove parole, le nuove regole grammaticali (Milici, 2019). Si crea un'atmosfera che si avvicina maggiormente alle conversazioni spontanee e che permette un uso autentico della lingua: i partecipanti modificano l'andamento della conversazione, tutti hanno diritto di prendere parola, sono frequenti le partenze simultanee, il contenuto dei turni non è stabilito in anticipo e tutti possono proporre ampliamenti tematici.

La presente ricerca si concentra su una specifica forma teatrale: il teatro sociale. Questa pratica, descritta brevemente nel paragrafo 3.2, aggiunge alla glottodidattica teatrale un lavoro di integrazione civica attraverso la condivisione delle proprie esperienze di vita, le proprie ambizioni e desideri, esplorati teatralmente attraverso improvvisazioni ed esercizi (Salza, 2017).

\subsection{Una definizione di teatro sociale}

Il teatro sociale è una pratica che «si occupa dell'espressione, della formazione e dell'interazione di persone, gruppi e comunità, attraverso attività performative che includono i diversi generi teatrali, il gioco, la festa, il rito, lo sport, il ballo» (Bernardi, 1998: 157). Gli obiettivi del teatro sociale sono di natura sociopolitica ed educativa: portare 
l'individuo a esplorare il proprio mondo interiore al fine di autoaffermarsi, autorappresentarsi e reinserirsi nella vita sociale e comunitaria (Pontremoli, 2005). I laboratori di teatro sociale si concentrano infatti nei luoghi in cui il soggetto non può esprimersi perché oppresso dalle condizioni: carceri, ospedali psichiatrici, comunità di recupero e isolamento di periferia (ibidem).

Le esperienze di teatro sociale operano in molteplici ambiti: esperienze generazionali, esperienze di genere, discriminazione sessuale, marginalità, che può essere di tipo economico sociale, detentiva, etnica o politica e infine esperienze di comunità periferiche (Dragone, 2000). I soggetti che promuovono percorsi teatrali di questo tipo si propongono come mezzi di costruzione, cura e formazione delle comunità, offrendo la possibilità di denuncia e protesta verso le situazioni di disagio, ma soprattutto di affermazione della propria identità (ibidem).

Le attività performative attraverso cui si intende raggiungere tali obiettivi sono il gioco, la danza e la commistione di diversi generi teatrali. Attraverso l'adozione di pratiche comunicative, espressive e relazionali il teatro offre ai partecipanti la possibilità di esprimere il proprio sé e le proprie emozioni all'interno di un contesto protetto, come quello del laboratorio. Inoltre, all'interno del gruppo che vive l'esperienza teatrale si crea uno spazio di condivisione e ascolto.

La rappresentazione scenica viene proposta solamente se il gruppo sente la necessità di aprirsi all'esterno. In questo caso lo spettacolo si costruirà sulla base di quanto emerso durante gli incontri e, per questo, ciò che si porterà sul palcoscenico sarà sempre concordato fra i partecipanti e i conduttori (Pontremoli, 2005).

\subsection{Le fasi del teatro sociale}

L'azione del teatro sociale si realizza in fasi precise: il training psicofisico, il training relazionale e l'esplorazione drammaturgica.

Il training psicofisico è centrato sulla scoperta del corpo come strumento di espressione, di comunicazione e di relazione. Durante questa fase i partecipanti esplorano il corpo, in relazione a sé, allo spazio, agli altri corpi e agli oggetti. Si sperimentano quindi la voce (nelle sue possibilità: urlando, parlando sottovoce, cantando), la mimica facciale, la respirazione, lo sguardo, la camminata, la corsa. Si mettono in gioco le possibili posture che il corpo assume durante lo svolgimento di ognuna di queste attività. Si lavora inoltre sulla scoperta dei segmenti del corpo e sulle possibilità delle diverse articolazioni: il movimento dei piedi, delle gambe, delle mani, del collo e del viso. Si sperimenta inoltre la camminata quotidiana per mettere in luce le andature, la velocità, la postura di ciascuno.

La fase del training relazionale è finalizzata alla formazione di un gruppo affiatato attraverso giochi ed esercizi che stimolano la fiducia e le dinamiche del conflitto. Nel contesto di fiducia e di assenza di giudizio il singolo sceglierà di aprirsi alla comunità, portando i propri vissuti personali. Le tecniche con cui si arriva a costruire questo clima di intesa sono molteplici e vengono inserite gradualmente durante il corso del laboratorio. Il contatto con l'altro si costruisce poco alla volta, partendo dall'esplorazione degli sguardi, fino ad arrivare al contatto fisico fra i partecipanti e alle sue possibilità di gioco, ballo, sostegno e affetto. Per sviluppare la fiducia si adottano tecniche tipiche dei laboratori teatrali: ad esempio lasciarsi cadere a occhi chiusi e poi farsi sorreggere da un compagno o dal gruppo; camminare a occhi bendati, seguendo le istruzioni vocali di un partecipante. Questa fase del laboratorio esplora inoltre gli aspetti negativi della relazione con l'altro, come il rifiuto, simulazioni di violenza, oppressione, attacco e difesa. 
L'ultima fase è quella dell'esplorazione drammaturgica e della costruzione scenica. Questa viene sempre elaborata, discussa e creata dal gruppo di partecipanti e dal conduttore. Lo spettacolo teatrale diventa un mezzo per aprirsi allo sguardo della comunità, per autorappresentare e rileggere il proprio vissuto e, grazie alla forza della coralità, di raccontare ciò che da soli non si avrebbe il coraggio di esprimere. Durante la fase laboratoriale il conduttore deve sia avere uno sguardo e un ascolto attivo rispetto a quanto accade, sia cogliere cosa possa essere inserito nello spettacolo. È necessario, infatti, che tutti i partecipanti abbiano la possibilità di avere un ruolo, di sentirsi rappresentati e di essere presenti sul palcoscenico con quanto hanno condiviso in fase di laboratorio (Bernardi, 1998; Dragone, 2000; Salza, 2017; Pontremoli, 2005).

\section{LA RICERCA EMPIRICA:}

OSSERVARE L'USO DEL TEATRO SOCIALE NELLA GLOTTODIDATTICA

\subsection{Obiettivo}

La presente ricerca, di tipo qualitativo, ha come obiettivo l'osservazione della partecipazione e delle dinamiche di interazione nel corso di due laboratori di teatro sociale rivolti a gruppi eterogenei di apprendenti migranti: il teatro sociale stimola la partecipazione alla conversazione in gruppi eterogenei? Se sì, quali dinamiche interazionali si possono osservare?

\subsection{Il contesto della ricerca}

La ricerca si inserisce all'interno di due percorsi differenti: il progetto La Danza delle Parole, ideato e condotto dalla compagnia Onda Teatro in collaborazione con la scuola di italiano per persone migranti del Sermig di Torino ${ }^{4}$ e il progetto Melting Pot, promosso dal Centro Interculturale ${ }^{5}$ del Comune di Torino e dalla Fondazione Teatro ragazzi e Giovani Onlus.

Il primo laboratorio osservato (da qui in avanti Lab1) è stato realizzato nei mesi di febbraio e marzo 2018, con cadenza bisettimanale, per un totale di 15 incontri della durata di due ore e mezza ciascuno e ha avuto come conclusione la messa in scena di due spettacoli aperti al pubblico: il primo presso il Sermig e il secondo presso la Casa del Teatro Ragazzi e Giovani di Torino. Il laboratorio, attivo dall'anno scolastico 2013/2014, è ideato e condotto dalle registe Francesca Guglielmino e Irene Salza, in collaborazione con i maestri volontari e con i coordinatori della scuola di italiano. Il laboratorio si è svolto nell'orario e nelle sedi consuete delle lezioni di italiano promosse dal Sermig.

Il secondo laboratorio osservato (da qui in avanti Lab2) si è svolto nei mesi di aprile, maggio e giugno 2018, per un totale di 12 incontri con cadenza settimanale e da due ore ciascuno. Il laboratorio rientrava nei servizi offerti dal Progetto Melting Pot, articolato in

\footnotetext{
${ }^{4}$ Il Sermig è un'organizzazione no-profit fondata da Ernesto Olivero e operativa nella città di Torino e in diversi Paesi del mondo dal 1964. Nella città di Torino offre svariati servizi, come la scuola di italiano per persone migranti, l'accoglienza per donne vittime di tratta, dormitori per persone senza fissa dimora, doposcuola per i bambini del quartiere di Porta Palazzo. Per maggiori informazioni: https://www.sermig.org/arsenali/arsenale-della-pace.html.

${ }^{5}$ Il Centro Interculturale della Città di Torino nasce nel 1996 con l'intento di offrire ai cittadini opportunità di formazione, occasioni di incontro e dialogo. Per maggiori informazioni sul Centro Interculturale della Città di Torino si veda il sito http://www.interculturatorino.it/.
} 
due laboratori (teatro sociale e danza terapia) volti a favorire l'accoglienza e l'inclusione dei richiedenti asilo o titolari di protezione internazionale o umanitaria inseriti nel Sistema di Protezione per Richiedenti Asilo e Rifugiati (SPRAR) ${ }^{6}$ messo in atto dal Servizio Stranieri della Direzione Politiche Sociali del Comune di Torino. I due laboratori sono stati proposti ai partecipanti come percorsi indipendenti, collegati da obiettivi comuni, che mirano alla valorizzazione dei bisogni linguistici ed espressivi dei gruppi, strettamente legati ai bisogni di inclusione socio-culturale. Il progetto si è concluso il 20 giugno 2018, in occasione della Giornata Mondiale del Rifugiato, attraverso la messa in scena di uno spettacolo di danza terapia e di uno spettacolo di teatro. Il progetto teatrale si è svolto anche in questo caso sotto la conduzione e l'ideazione di Francesca Guglielmino e Irene Salza, le quali sono state affiancate da un terzo regista, Alberto Valente.

\subsection{I principi organizzativi dei due laboratori}

I due laboratori osservati rispondono ai medesimi principi organizzativi, nonostante si siano svolti in luoghi e in contesti differenti: il teatro come possibilità di espressione e di rappresentazione sia della persona sia del gruppo. Il lavoro proposto ha soprattutto l'intento di valorizzare l'esperienza di vita dei partecipanti, la promozione del dialogo critico fra i componenti del gruppo e il raggiungimento di una competenza fluida in lingua seconda, che permetta al singolo di definirsi, esprimere sé stesso e la propria cultura. La messa in scena di uno spettacolo teatrale, aperto al pubblico, ha inoltre la funzione di permettere ai partecipanti di vivere un'esperienza trasformativa: le proprie storie, canzoni e danze portate in scena permettono sia di offrire al pubblico l'esperienza vissuta sia di vedere affermata la propria persona.

Entrambi i progetti pongono l'attenzione sul consolidamento degli aspetti linguistici e comunicativi della lingua italiana:

le strutture linguistiche e gli elementi lessicali trattati emergono chiaramente (...) quali assi portanti della costruzione drammaturgica di ogni performance, oltre che come ponti per creare collegamenti tra le proprie lingue e culture di origine e quella del paese di accoglienza (Salza, 2017: 119).

\subsection{Partecipanti}

Presenteremo coloro che hanno preso parte ai due laboratori di teatro sociale in base al laboratorio da loro frequentato. Per ciascun gruppo verrà presentato un quadro generale per mettere in luce l'eterogeneità che caratterizza i partecipanti per quel che riguarda il genere, l'età, la provenienza, la lingua madre, la data di arrivo in Italia, il grado di istruzione e la tipologia di documenti.

\footnotetext{
${ }^{6}$ Il progetto S.P.R.A.R. si occupa dell'accoglienza beneficiari/e Richiedenti Asilo e titolari di Protezione internazionale, inseriti in percorsi di accoglienza e integrazione sul territorio cittadino. Per maggiori informazioni sul progetto S.P.R.A.R. si veda il sito internet https://www.sprar.it.

${ }^{7}$ Per la stesura di questo paragrafo sono stati usati e consultati i materiali di lavoro (progetti, appunti) della regista teatrale e dottoranda (Universidad Autònoma di Barcellona) Irene Salza, la quale ha condotto, insieme a Francesca Guglielmino e ad Alberto Valente, i laboratori teatrali presi in esame della presente ricerca.
} 


\subsubsection{Il gruppo del Lab1}

Il gruppo è composto da 20 persone, di cui 14 donne e 6 uomini. L'età è compresa fra i 21 anni e i 56 anni, tuttavia la maggior parte dei partecipanti ha meno di 25 anni. I Paesi di provenienza sono: il Mali (una persona), la Costa d'Avorio (quattro persone), il Togo (una persona), il Camerun (una persona), la Nigeria (undici persone), l'Armenia (una persona) e la Cina (una persona). Le L1 dei partecipanti sono: l'inglese, il malinke, il francese, il bambarà, il cotocoli, l'edo, il cinese, l'armeno, il russo e il tedesco. Tredici partecipanti sono arrivati in Italia fra il 2016 e il 2017, una persona nel 2015, due nel 2014, una nel 2012, due nel 2010 e una nel 2005. Quattordici persone sono richiedenti asilo, cinque persone non hanno $i$ documenti e una persona ha $i$ documenti per ricongiungimento familiare. Per quel che riguarda il grado di istruzione, invece, 6 persone non hanno frequentato la scuola nel proprio Paese di origine, 3 persone hanno frequentato la scuola primaria, 3 persone hanno la licenzia media, 6 persone hanno frequentato la scuola superiore e 2 persone sono laureate.

\subsubsection{Il gruppo del Lab2}

Il gruppo è composto da 14 persone ospitate in differenti progetti di accoglienza, molti di loro si sono infatti incontrati per la prima volta in questa sede. Il gruppo è composto da 3 donne e da 11 uomini, quasi tutti i partecipanti sono richiedenti asilo (due hanno ottenuto lo status di rifugiato politico). I Paesi di provenienza sono sette: la Guinea (due persone), la Nigeria (quattro persone), il Venezuela, il Congo, il Mali (quattro persone), il Gambia e la Palestina. Le L1 sono: l'edo, l'inglese, l'arabo, il bambarà, il fula, il francese, il mandinga, lo spagnolo, il kikongo e il wolof. Le date di arrivo in Italia si concentrano nel 2017, 2016, 2015 e 2014, una persona è arrivata nel 2005. Per quel che riguarda il livello di scolarizzazione tre persone risultano non aver studiato nel proprio Paese, tre persone hanno frequentato la scuola primaria, quattro persone la scuola media e tre la scuola superiore.

\subsection{Criteri e strumenti di analisi}

Gli incontri dei due laboratori sono stati registrati in formato audio-video e accompagnati dalla stesura di un diario di campo, al fine di ricostruirne il contesto sia attraverso la visione dei video sia attraverso la consultazione delle note personali ${ }^{8}$. Il materiale raccolto è stato poi analizzato e parte delle conversazioni ritenute più rilevanti sono state trascritte tramite il programma SayMore 3.0. 243. I criteri di annotazione delle

\footnotetext{
${ }^{8} \mathrm{Al}$ fine di poter procedere con la ricerca, che ha previsto sia il trattamento dei dati sensibili dei partecipanti sia la ripresa audio-video dell'intero laboratorio, ci siamo premurati di indirizzare una lettera di autorizzazione rivolta al Centro Interculturale, al Servizio Stranieri della città di Torino e alla Fondazione del Teatro Ragazzi. Inoltre, abbiamo fornito ai partecipanti una liberatoria per effettuare le riprese e per usare il materiale raccolto allo scopo della nostra ricerca. Per assicurarci la completa comprensione del documento ci siamo premurati di scrivere il testo usando un linguaggio chiaro e semplice. In entrambi i laboratori i partecipanti sono stati informati della ricerca da me condotta, nel primo caso la mia figura coincideva con quella dell'insegnante di italiano, mentre nel secondo caso rappresentava un elemento nuovo per il gruppo. In entrambi i laboratori ho preso parte attivamente a tutte le fasi del progetto, inclusa quella performativa.
} 
trascrizioni si basano sulle convenzioni MacWhinney (1995). Le trascrizioni delle attività sono successivamente state analizzate secondo i parametri dell'Analisi della Conversazione, seguendo gli studi di Sacks, Schegloff e Jefferson (1974), Sacks (2007), Bazzanella (1994), Orletti (2000), Fele (2007), Kunitz (2019).

\subsection{Ambiti di analisi}

Al fine di mettere in luce le caratteristiche dei fenomeni comunicativi sono stati individuati due ambiti di analisi: le forme della turnazione e il contenuto dei turni. Nei sottoparagrafi 5.1 e 5.2 si presenterà una esemplificazione dei turni ritenuti più rilevanti per ciascun ambito di analisi. Nel paragrafo 6 verrà presentata l'analisi dei risultati, individuando delle scale su cui qualificare i tipi di turni individuati e presentando gli indicatori di verifica che mettono in luce il coinvolgimento dei partecipanti a intervenire nella conversazione.

Per quel che riguarda le forme della turnazione ci siamo soffermati sulle auto-selezioni, in quanto fenomeno quasi del tutto assente nelle conversazioni asimmetriche. Nello specifico, abbiamo individuato due tipologie di autoselezione:

a) le autoselezioni richieste dalla regia (l'apprendente prende parola per rispondere a una domanda dell'insegnante rivolta al gruppo intero o a uno specifico partecipante);

b) le autoselezioni non richieste dalla regia (i partecipanti prendono parola spontaneamente per commentare il turno di un pari o di un regista, per tradurre il turno di un altro partecipante e per raccontare alla classe un episodio al posto di un altro partecipante).

La seconda categoria di analisi riguarda invece il contenuto dei turni, in questo caso ci siamo soffermati su due fenomeni:

a) contenuto vicino all'interazione didattica, ossia i turni che dimostrano una certa attenzione alla forma linguistica (le autocorrezioni e le eterocorrezioni);

b) contenuto distante dall'interazione didattica, ossia la condivisione di aneddoti personali, i commenti sul contenuto del turno di un pari, la partecipazione emotiva (battute, scherzi fra pari o con i registi).

\section{ESEMPLIFICAZIONE DEI TURNI PIÙ SIGNIFICATIVI}

\subsection{Prima categoria di analisi: le forme della turnarione}

L'esempio scelto per descrivere le forme della turnazione riguarda il secondo fenomeno: le autoselezioni non richieste dalla regia. La scena selezionata si è svolta il sesto giorno del Lab1. Le registe, dopo aver illustrato il concetto di "talento", hanno invitato i partecipanti a condividere con il gruppo un proprio talento, capacità o passione: una partecipante ha parlato della propria abilità nel piccolo artigianato e della propria produzione artistica (dipinti, bigiotteria, lavori all'uncinetto); un'altra ha condiviso la propria passione per la sartoria e un terzo partecipante ha tradotto dal bambarà il racconto di un compagno riguardo la coltivazione del cacao. 
(C) Italiano LinguaDue 2. 2021. C. Canuto, Stimolare la partecipazione alla conversazione in classi con livello eterogeneo di scolarizzazione. Le pratiche del teatro sociale per sviluppare scopi comunicativi reali: l'espressione di sé e l'integrazione

Nel frammento proposto troviamo la studentessa IND $^{9}$ intenta a condividere il proprio talento come elettricista. La partecipante ha difficoltà nell'esporre il proprio mestiere e il resto del gruppo non comprende pienamente il messaggio. La scarsa competenza comunicativa della studentessa porta però a una particolare situazione: $i$ partecipanti, senza essere sollecitati dalle registe, mostrano un forte interesse a capire il messaggio di IND, proponendo domande e interpretazioni. Queste mosse conversazionali sono significative in quanto non previste da parte di chi conduce l'attività. Possiamo infatti osservare come per dieci turni (dal T04 al T13) la conversazione sia interamente gestita da tre partecipanti (IND, BEL e ADA) anziché dalle registe o dall'insegnante di lingua. I due uomini $\left(\mathrm{BEL}^{10}\right.$ e $\mathrm{ADA}^{11}$ ) inizialmente (T05 e T07) si autoselezionano per spiegare il tipo di macchina usata da IND e successivamente per rivolgere alla compagna alcune domande (T08). In particolare, il coinvolgimento di BEL si può rilevare dal modo in cui interpreta i messaggi di IND, arrivando persino a contraddirla ("allora radiatore") e successivamente a correggerla ("no elettricità eh").

\section{Esempio 1}

T01*IND: elettricista!

T02*IRE: elettricista?

T03*IRE: uao!

T04*IND: facevo elettricista \# faccio \#\# ehm macchina-, macchina

T05*BEL: macchina elettricità!

T06*IND: eh sï\# acqua

T07*JON: elettricità macchina

T08*BEL: acqua?

T09*IND: sì

T10*BEL: $\quad$ allora radiatore quando\#

T11*IRE: acqua?

T12*IND: sì per macchina

T13*BEL: no elettricità eh

Qualche turno successivo (T26 e T28) si inserisce inoltre LEY ${ }^{12}$ la quale rivolge ben tre domande alla compagna ("Come macchina? Macchina per fare cosa? Lavoravi in azienda?"). La potenza di tale turno risiede nel fatto che solitamente il fare domande sia una prerogativa degli insegnanti e non degli studenti (Fele 2003).

T24*IND: in Cina\# Cina 5 anni fa facevo l'elettricista di macchina

T25*FRA: ok

T26*LEY: come macchina? macchina per fare cosa?

T27*IND: macchina eh senti\# acqua

T28*LEY: lavoravi in azienda?

T29*BEL: radiateur

9 IND: originaria della Cina; anno di arrivo in Italia: 2016; documenti: ricongiungimento familiare; scolarizzazione: diploma.

${ }^{10}$ BEL: originario del Mali; anno di arrivo in Italia: 2014; documenti: nessuno; scolarizzazione: nessuno.

11 ADA: orinario della Costa d'Avorio; anno di arrivo in Italia: 2016; documenti: richiedente asilo; scolarizzazione: scuola primaria.

${ }^{12}$ LEY: originaria dell'Armenia; anno di arrivo in Italia: 2017; documenti: richiedente asilo; scolarizzazione: laurea. 
(C) Italiano LinguaDue 2. 2021. C. Canuto, Stimolare la partecipazione alla conversazione in classi con livello eterogeneo di scolarizzazione. Le pratiche del teatro sociale per sviluppare scopi comunicativi reali: l'espressione di sé e l'integrazione

T30*JON: radiateur

T31*ADA: radiateur

T32*DAU: radiateur

T33*BEL: radiateur

T34*LEY: credo che azienda?

T35*FRA: dici che è il radiatore? (\%ridendo)

T36*ADA: radiateur

T37*FRA: radiatore. avete fatto un coro eh! radiateur\# radiateur!

(\%risate)

T38*BEL: no quello lavoro radiatore-, non è elettricità

T39*FRA: eh infatti\# non so se\# ok faceva funzionare dei\# cioè tu lavoravi a delle macchine con l'acqua?

T40*BEL: no, no macchina grande! sì!

T41*CLA: ma lo saprà lei (\%ridendo)

A rispondere alle domande di LEY però non è IND, bensì quattro partecipanti (T29T33"'Radiateur! Radiateur! Radiateur!") che in coro sostengono che la compagna di professione aggiustasse radiatori. LEY si aggiunge ai commenti proponendo un'ossevazione in toni dubitativi ("credo che azienda?"), la regista FRA commenta ridendo la reazione dei quattro partecipanti (T37) a cui rispondono ancora ADA e BEL (ormai protagonista della conversazione) per sostenere la loro teoria ("no quello lavoro radiatore-, non è elettricità"). Questi turni mostrano dunque un'alta partecipazione da parte dei partecipanti i quali, a causa del basso livello della compagna, non riescono a capire alcuni dettagli del racconto ma si mostrano fortemente motivati a capire la situazione. Inoltre, il fatto che IND sia l'unica partecipante sinofona non permette l'uso della traduzione per raggiungere l'obiettivo comunicativo. Queste dinamiche permettono di rompere lo schema tipico della conversazione in classe, che vede gli insegnanti protagonisti e gestori dei turni, e permettono di avvicinarsi alle dinamiche della conversazione ordinaria: sono gli apprendenti ad avere il potere della conversazione, gareggiando fra loro per accedere ai turni di parola e creandosi uno spazio di parola più ampio e personalizzato rispetto a quanto solitamente accade in una classe di lingua (Orletti, 2000).

\subsection{Seconda categoria di analisi: il contenuto del turno}

Per quel che riguarda il contenuto del turno abbiamo raccolto numerose eterocorrezioni e autocorrezioni, in cui i partecipanti hanno dimostrato una certa attenzione alla forma degli enunciati. Le eterocorrezioni non avvengono frequentemente in una classe di lingua: le correzioni degli insegnanti sono accettate e rispettate, le correzioni fra pari necessitano di un certo stato emotivo perché «si è imbarazzati, incerti della propria competenza» (Nuzzo, Grassi, 2016: 157). Si può essere restii nella proposta o nell'accettazione di correzioni fra pari: la costruzione di un clima positivo e collaborativo che «accolga con entusiasmo le proposte di attività fra pari non va dunque data per scontata» (ibidem). 
Nell'esempio che segue troviamo la classe ${ }^{13}$ intenta a rappresentare una scuola di italiano L2. Una studentessa, YUU ${ }^{14}$, si offre di fare l'insegnante e inscena il primo giorno di scuola: si presenta e chiede a ogni studente di spiegare il motivo per cui si sono iscritti alla scuola di italiano. Vediamo YUU (ancora nei panni della maestra di italiano) selezionare $\mathrm{MAR}^{15}$ per chiedere anche a lei come mai voglia imparare la lingua italiana. E interessante ribadire come questa iniziativa legata alla domanda "perché vuoi imparare l'italiano?" sia nata spontaneamente da YUU. La richiesta delle registe era di fingere di essere in classe, lasciando la possibilità agli studenti di gestire liberamente l'attività. Mentre MAR sta elaborando la propria risposta (T02), YUU si autoseleziona per correggere l'uso di 'io piace' in 'mi piace' (T03). La classe scoppia a ridere, ma la regista interviene subito per invitare YUU a non correggere i compagni (T05), mostrando di non cedere davvero a YUU il ruolo di insegnante. MAR coglie la correzione, ripetuta fra l'altro nel turno della regista, e ripete l'elemento candidato da YUU.

\section{Esempio 2}

T01*YUU: e tu signorina, escusa, signorina\# e\#e come si chiama? eh vorrei sentire la signorina! lei è assieme qua e\# prima due anni che è con me nella nostra scuola-, voleva sentite-, perché tu vuoi studiare italiano? (\%indicando MAR)

T02*MAR: perché io vuoi studiare italiano, io piace italiano, io piace tua lingua\# io voglio parlare bene..+

T03*YUU: a me! a me! a me piace tua lingua! (\%risate)

T04*IRE: aspetta YUU non ti preoccupare adesso di correggerli-, va bene-, a me piace e\# e poi?

T05*MAR: a me piace loro lingua, io voglio parlare bene

Il terzo esempio proposto è particolarmente interessante per due ragioni: il contenuto strettamente personale del turno e il ricorso spontaneo alla traduzione in L2 da parte di un altro partecipante. Il quarto e il quinto giorno del Lab1 le conduttrici propongono un'attività sul significato del concetto di "coraggio". Le registe invitano la classe a riflettere su quale possa essere una persona o un personaggio per loro coraggioso. Due uomini si selezionano per primi e raccontano aneddoti personali: quando si va a scuola per la prima volta, quando si fa qualcosa di nuovo. IND decide di portare sé stessa come esempio di coraggio, raccontando la propria storia di migrazione. Questo condizionerà la maggior parte dei partecipanti, i quali sceglieranno di condividere aneddoti personali legati a un momento in cui hanno agito con coraggio. Per quel che riguarda il secondo aspetto di interesse, l'uso della traduzione, nel corso dei due laboratori di teatro molto frequentemente si è ricorso all'uso della traduzione per coinvolgere i partecipanti con minore competenza linguistica. Il più delle volte, lo strumento della traduzione veniva usato su sollecitazione dei registi, i quali o chiedevano espressamente a un partecipante di tradurre il turno dalla lingua madre del parlante all'italiano, oppure chiedevano al parlante di esprimersi, usando una lingua veicolare e si facevano loro stessi traduttori. L'uso della traduzione è una delle manifestazioni della pedagogia del teatro sociale, che intende

\footnotetext{
13 Settimo giorno del laboratorio Lab1.

14 YUU: originaria della Costa d'Avorio; anno di arrivo in Italia: 2005; documenti: nessuno; livello di scolarizzazione: scuola superiore.

${ }^{15}$ MAR: originaria della Nigeria; anno di arrivo in Italia: 2016; documenti: nessuno; livello di scolarizzazione: scuola primaria.
} 
mantenere vivo l'obiettivo comunicativo esplorando diverse possibilità per permettere ai partecipanti di esprimere la propria persona.

Nel frammento che segue vediamo VIC ${ }^{16}$ intenta a condividere con la classe un esempio di coraggio. L'esitazione e l'incertezza di VIC sono messi in luce sia a livello prosodico (il tono della voce di VIC è bassissimo e parlerà sottovoce per tutta la durata della scena) sia dalle numerose pause che caratterizzano il primo turno. La compagna $\mathrm{BEN}^{17}$ si autoseleziona in una pausa di VIC per spiegare al resto della classe quanto detto da VIC nel T1. Questo tipo di turno solitamente si riscontra nelle interazioni istituzionali, in cui la figura che detiene il potere sintetizza con formulazioni di riepilogo il contenuto del turno precedente (Orletti, 2000). Il fatto che in questo caso, invece, sia stata BEN ad autoselezionarsi per riformulare il concetto della compagna mostra come il coinvolgimento possa portare un'apprendente ad assumersi un ruolo di regia. BEN va quindi in soccorso della compagna e da quel momento in poi VIC si rivolgerà a lei, sottovoce e in pidgin english, e BEN si assumerà il ruolo di traduttrice per il resto della conversazione. Il coinvolgimento di BEN verso l'attività è inoltre evidenziato al T12 in cui la regista formula una domanda di chiarimento verso VIC e a rispondere saranno entrambe le studentesse in coro.

\section{Esempio 3}

T01*VIC: $\quad$ si\# per me\# io\# iniziato\# casa (\%mentre parla ha una mano nella tasca della felpa di BEN)

(\%GAG fa segno di alzare la voce)

$\square \mathrm{T} 02 * \mathrm{BEN}$ : eh\# una persona che aiuta loro a casa

T03*VIC: (\%parla a BEN sottovoce in pidgin English)

T04*BEN: loro sempre dire lei\# non ti preoccupare andrà tutto bene

T05*VIC: (\%parla a BEN sottovoce in pidgin English)

T06*BEN: ah ok... un giorno parlerai questo lingua\# italiano

T07*FRA: ah ok

T08 *VIC: (parla a BEN sottovoce in pidgin English)

T09*BEN: perché lei ha provato lavoro\# non puoi lavorare perché non sai parlare

T10*FRA: una persona che ti dà coraggio\# qualcuno che ti dà coraggio... giusto? quị?

T11*VIC: si!

T12*BEN: sì! (\%in coro)

T13*FRA: ok

Il frammento che segue manifesta l'intento del teatro sociale di esplorare diverse possibilità per dare voce a tutti i partecipanti. La scena si apre con le due registe FRA e IRE che sollecitano i partecipanti a condividere un proprio esempio di coraggio. Quasi tutti gli apprendenti hanno eseguito la richiesta, eccetto $\mathrm{AIK}^{18}$ e $^{\mathrm{GLO}}{ }^{19}$, rispettivamente di un livello pre A1 e A0. Al T2, innanzitutto, vediamo AIK rifiutarsi di eseguire la

\footnotetext{
16 VIC: originaria della Nigeria; anno di arrivo in Italia: 2017; documenti: richiedente asilo; livello di scolarizzazione: scuola primaria.

17 BEN: originaria della Nigeria; anno di arrivo in Italia: 2016; documenti: richiedente asilo; livello di scolarizzazione: scuola superiore.

18 AIK: originaria della Nigeria; anno di arrivo in Italia: 2016; documenti: richiedente asilo; livello di scolarizzazione: nessuno.

19 GLO: originaria della Nigeria; anno di arrivo in Italia: 2017; documenti: asilo politico; livello di scolarizzazione: nessuno.
} 
richiesta della regista, fatto sicuramente eccezionale in quanto rappresenta un rovesciamento dei ruoli sociali tipici dell'interazione in classe e che infatti suscita l'ilarità fra i compagni. Al T07 vediamo YUU auto-selezionarsi per condividere un esempio di coraggio, senza ricevere sollecitazioni dirette dalle registe. E infatti importante sottolineare che il giorno precedente YUU aveva già portato il proprio contributo all'attività. Tuttavia, YUU si autoseleziona per rendere protagonista del racconto la compagna AIK, che poco prima si era rifiutata di partecipare. Riteniamo che questa mossa sia particolarmente significativa n quanto mette in luce la collaborazione fra pari e il desiderio di coinvolgere i propri compagni nelle attività. YUU racconta quindi un episodio in cui la compagna AIK è stata per lei un esempio di coraggio: le due donne era ospiti nello stesso dormitorio e AIK non dormiva da giorni perché sua figlia $\mathrm{SAB}$, neonata, aveva male alla pancia.

Un dato interessante, che mostra la distanza dell'interazione della scena da quelle dell'ambito scolastico, risiede nella durata del turno di YUU. Infatti, la dominanza quantitativa (Orletti, 2000), intesa come la durata del turno di parola, è uno dei tipi di dominanza che segnalano le disparità nei diritti conversazionali. L'ultimo elemento che segnaliamo, in quanto si avvicina al contesto della conversazione naturale, è la reazione delle registe: entrambe risponderanno portando delle considerazioni di contenuto e non di forma, entrando in empatia fra l'altro con la condizione di AIK (T12: "tu sei molto coraggiosa" e T13: "le mamme che non dormono sono molto coraggiose! sì, capisco bene!").

\section{Esempio 4}

T01*FRA: poi? se qualcuno ha ancora voglia... se no

T02*AIK: no! (\%risate della classe)

T03*IRE: anche solo dire la persona che ti ha ispirato, nient'altro! per esempio\# una persona coraggiosa è\#\# il nome

T04*BEN: $\quad$ SAB

T05*FRA: SAB (\%ridendo)

T06*IRE: $\quad$ SAB\# è vero! (\%ridendo)

T07*YUU: la mamma di SAB è coraggiosa

T08*IRE: eh sï\# eh!

T09*YUU: io dormito con lei due giorni (\% risate)

T10*YUU: la sua bambina dorm\# non dormiva\# lei no\# SAB aveva un settimane\# non dorme e piange piange piange\# tutte la notte tutt\# noi stavamo dormendo\# lei poverina\# seduta così fino a mattina\# e lei non dormiva un giorno\# due giorni, un giorno ho detto lei non ti fa dormire? detto sì! ma aspetta! io sono mamma faccio vedere ho preso SAB e l'ho bagnata, ha dormito\# quella notte abbiamo dormito insieme\# lei no dormiva! Ha fatto un mese! lei non dormiva con la bambina

T11*FRA: coraggiosa!

T12*IRE: $\quad$ tu sei molto coraggiosa (\%guardando AIK)

T13*FRA: le mamme che non dormono sono molto coraggiose! sì, capisco bene! 


\section{ANALISI DEI RISULTATI}

In questa sede ci occuperemo di discutere le caratteristiche dei fenomeni comunicativi raccolti, e in parte presentati nel paragrafo precedente, al fine di rendere espliciti i fattori che mettono in luce il coinvolgimento del gruppo a partecipare alla conversazione nel corso dei due laboratori. Inizialmente osserveremo $i$ dati forniti dalle trascrizioni per concentrarci sugli elementi su cui si basa la nostra analisi: la gestione dei turni delle conversazioni e il loro contenuto. I dati verranno confrontati con il tipo di attività in cui si presentano tali fenomeni. In secondo luogo, ci soffermeremo sul ruolo delle mosse dei registi, promosse dalla metodologia del Teatro Sociale, rivelandone le conseguenze che generano sull'interazione del gruppo.

\subsection{Parametri rilevati per l'individuazione del coinvolgimento dei partecipanti nella conversazione}

Attraverso l'analisi delle conversazioni trascritte, è stato possibile individuare alcuni indicatori del coinvolgimento dei partecipanti nella conversazione, facendo riferimento alle classificazioni proposte da Balboni (2015) e Pallotti (2012) secondo cui le motivazioni più potenti del contesto classe sono strettamente connesse alle dinamiche di gruppo, alla figura del docente e alle attività usate. Come anticipato nel sottoparagrafo 4.6, gli indicatori che abbiamo individuato riguardano la presa del turno nel corso delle conversazioni, in particolare le autoselezioni. Abbiamo quindi classificato tali indicatori in base al valore della loro forza interazionale, che risiede nella possibilità di portare modifiche nell'organizzazione dello scambio comunicativo tipico della classe (Orletti, 2000). Il coinvolgimento dei partecipanti è evidenziato dalla forza dei loro turni all'interno della conversazione. Proveremo quindi a presentare in uno schema le forme della turnazione raccolte presentandole come un continuum, in cui all'estrema sinistra troviamo le mosse dal grado più debole (-) e all'estrema destra le mosse dal grado più forte $(+)$ :

\section{Continuum autoselezioni}

Autoselezioni richieste dalla regia Autoselezioni non richieste dalla regia

All'estrema sinistra troviamo le autoselezioni richieste dalla regia, ossia le autoselezioni sollecitate dagli insegnanti o dai registi teatrali. Queste mosse sono poco significative perché previste e sono state registrate sia in giorni sia in attività differenti. Essendo tale tecnica di attribuzione del turno piuttosto frequente nel tipico contesto classe, non stupisce il fatto che l'autoselezione richiesta dalla regia non sia connessa al tipo di attività o a un giorno specifico del laboratorio.

Per quel che riguarda le mosse più forti, quindi quelle non richieste dalla regia, possiamo nuovamente presentare in uno schema la tipologia di autoselezione al fine di analizzare nel dettaglio il margine destro del continuum precedente ("continuum autoselezioni’’):

Continuum autoselezioni non richieste dalla regia

$\begin{array}{lllll}\text { ACP ACR } & \text { ATP } & \text { ACOP } & \text { AIR } & \text { ASR }\end{array}$


Anche in questo caso all'estrema sinistra troviamo le mosse più deboli: ACP (Autoselezioni per Commentare il turno di un Pari) e ACR (Autoselezioni per Commentare il turno di un Regista). A metà schema troviamo le mosse mediamente forti: ATP (Autoselezioni per Tradurre il turno di un Pari) e ACOP (Autoselezioni per Coinvolgere un Pari). All'estrema destra troviamo le autoselezioni più forti, in cui i partecipanti assumono il potere della conversazione: AIR (Autoselezioni per Interrompere un Regista) e ASR (Autoselezioni per Sostituirsi a un Regista). Per quel che riguarda le due mosse individuate come meno forti, ossia il commento al turno di un pari (ACP) e il commento al turno di un regista (ACR), il loro interesse risiede nel fatto che il fare commenti o domande, nelle interazioni in classe è prerogativa dell'insegnante (Fele, 2003) e non degli studenti. In questo modo viene a cadere lo schema tipico della conversazione in classe, che vede gli insegnanti protagonisti dell'azione e che commentano i turni degli apprendenti. Per quel che riguarda invece le autoselezioni per tradurre al posto di un pari (ATP) e per coinvolgere un pari (ACOP), la loro forza risiede nel carattere cooperativo, che mette in luce il clima di fiducia e collaborazione instauratosi nel gruppo. Infine, le autoselezioni per interrompere i registi (AIR) e per sostituirsi a essi (ASR) sono decisamente significative in quanto rappresentano una violazione delle norme di regia e l'assunzione del potere e del controllo conversazionale da parte dello studente, anziché dell'insegnante (Orletti, 2000).

A differenza delle autoselezioni richieste dalla regia, che abbiamo rilevato trasversalmente in numerose attività e in giorni differenti, in questo caso gli indicatori di coinvolgimento individuati si concentrano prevalentemente intorno al quarto, al quinto e al sesto giorno di laboratorio, ossia nella sua fase centrale, quella in cui i partecipanti hanno potuto sperimentare i propri vissuti attraverso il gioco teatrale e hanno avuto il tempo di costruire una relazione con il resto del gruppo. I campioni raccolti si concentrano infatti nelle attività che prevedono la possibilità di condividere con il resto del gruppo aneddoti personali, storie o canzoni significative, promuovendo il dialogo critico all'interno gruppo.

Ulteriori indicatori del coinvolgimento a partecipare alla conversazione risiedono nel contenuto dei turni delle interazioni, come anticipato nel sottoparagrafo 4.6: la condivisione del vissuto strettamente personale, l'interesse verso i turni dei pari e la partecipazione emotiva. La partecipazione emotiva è segnalata dalle numerose battute e scambi scherzosi, l'interesse verso il contenuto dei turni dei pari è segnalato dai numerosi commenti e riferimenti alle produzioni dei compagni, mentre la condivisione del vissuto personale si è spinta fino a sfiorare temi altamente intimi e portatori di un vissuto emotivo profondo. Molti partecipanti hanno infatti condiviso con il gruppo episodi legati alla propria storia di migrazione, segnata da momenti traumatici e di alta vulnerabilità, come il rischio di diventare vittima della tratta di esseri umani.

\subsection{I turni dei registi}

Gli indicatori di verifica più significativi che abbiamo registrato riguardano tuttavia le caratteristiche delle produzioni dei registi, i quali propongono una ridistribuzione del potere interazionale. Nel corso dei due laboratori di teatro sociale i registi hanno sovente rinunciato a più di un tipo di dominanza, ridistribuendo la simmetria nell'interazione.

I momenti in cui i registi hanno rinunciato alla dominanza quantitativa, misurata nella durata dei turni, nel numero di parole dette o nel numero dei turni, sono numerosi. Nella tabella riportiamo due esempi che mostrano i registi mentre si astengono dall'intervenire nelle interazioni, rinunciando a portare il proprio contributo e a portare correzioni. In questo modo i registi hanno permesso ai partecipanti di gestire l'interazione in autonomia, 
favorendone così uno sviluppo fluido e spontaneo. Nel primo esempio la classe è intenta a interpretare $\mathrm{i}$ gesti di un compagno, il quale sta mimando una scena della propria quotidianità: alla domanda della regista prendono parola cinque partecipanti differenti che cooperano per costruire il significato della scena mimata. Il secondo esempio (di cui riportiamo un breve estratto) mostra una partecipante intenta a rispondere a una domanda di una regista. Il frammento è significativo in quanto la partecipante produce una sorta di monologo costruendosi uno spazio di parola molto ampio, in cui nessuno la interromperà per diversi minuti.

Tabella 2.

\begin{tabular}{|c|c|}
\hline $\begin{array}{l}\text { Turni dei registi: } \\
\text { rinuncia alla dominanza } \\
\text { quantitativa }\end{array}$ & Esempi: \\
\hline & $\begin{array}{l}\text { Giorno } 4 \text { Lab } 2 \\
\text { T13*IRE: cosa c'era? cos'abbiamo visto? } \\
\text { T14*FAN: caldo e\# uhm } \\
\text { T15*LASS: c'è caldo, loro fanno la doccia } \\
\text { T16*GAG: doccia! } \\
\text { (\%qualcuno ride) } \\
\text { T17*FAN: c'è caldo, tu doccia\# tu prendi pantaloni } \\
\text { T18*YAY: e vestiti! } \\
\text { T19*FAN: e vestiti e e camicie! pur metti a } \\
\text { T20*MOM: doccia! (\%interrompe FAN) }\end{array}$ \\
\hline & $\begin{array}{l}\text { Giorno } 5 \text { Lab } 1 \\
\text { T06*EST: mio coraggio\# eh mia madre sempre coraggio\# mio } \\
\text { padre prima lavora poi loro\# ho detto stai a casa\# } \\
\text { anche lui-, molto\# sempre e non stai bene così-, c’è } \\
\text { malata! poi mia mamma sempre coraggio\# fai } \\
\text { mangiare per noi\# fai lavoro-, tanti lavoro per per } \\
\text { fare soldi\# poi io pensa che io cercare lavoro, finisce } \\
\text { scuola, vai lavoro\# ma non c’è lavoro mio paese! } \\
\text { (...) }\end{array}$ \\
\hline
\end{tabular}

Gli esempi che seguono riportano invece la rinuncia, da parte dei registi, alla dominanza interazionale, intesa come l'uso di mosse forti che controllano l'organizzazione dei turni. Abbiamo quindi riportato alcune situazioni in cui i partecipanti si sono autoselezionati per interrompere il turno di un regista. I registi si dimostrano disponibili ad accogliere le autoselezioni inaspettate e non richieste, offrendo feedback relativi al contenuto dei turni dei partecipanti, ignorando l'interruzione. 
Tabella 3.

\begin{tabular}{|l|l|}
\hline $\begin{array}{l}\text { Turni dei registi: } \\
\text { rinuncia alla dominanza } \\
\text { interazionale }\end{array}$ & Esempio: \\
\hline & $\begin{array}{l}\text { Giorno 4 Lab 1 } \\
\text { T02*BEL: coraggio per me è quando si è qualcosa-, non è } \\
\text { tuo\# sì paura e tu vai e prendi\# hai coraggio vai e } \\
\text { prendi-, (xx) allora se tu vai quando trovato } \\
\text { qualcosa che vuoi prendi, lui va e prendi (xx)... }+ \\
\text { (\%)olume molto basso) }\end{array}$ \\
& $\begin{array}{l}\text { T03*FRA: ok\# ah\# ah\#\# se hai coraggio puoi arrivare prima } \\
\text { da qualche parte e ottenere delle cose-, se non ce } \\
\text { l'hai puoi rischiare di rimanere indietro\# ok... bene! } \\
\text { E una parola che ha tanti significati }\end{array}$ \\
\hline
\end{tabular}

In un caso abbiamo rilevato la rinuncia da parte dei registi alla dominanza strategica: il regista $\mathrm{ALB}$, nel corso di un'attività relativa alla condivisione di canzoni, chiede indicazioni alla partecipante OSA sullo svolgimento dell'esercizio, portando quindi un rovesciamento dei ruoli.

Tabella 4.

\begin{tabular}{|l|l|}
\hline $\begin{array}{l}\text { Turni dei registi: } \\
\text { rinuncia alla dominanza } \\
\text { strategica }\end{array}$ & Esempio: \\
\hline & Giorno 6 Lab 2: \\
& $\begin{array}{l}\text { T04*ALB: ma possiamo fare il sottofondo, no? } \\
\text { T05*OSA: aspetta prima volta e dopo tu } \\
\end{array}$ \\
\hline & T06*ALB: ok ok \\
\hline
\end{tabular}

Un altro fattore significativo che caratterizza i turni dei registi e che stimola il coinvolgimento dei partecipanti nelle interazioni riguarda la forma del terzo turno. Gli esempi che seguono mostrano come il terzo turno dei registi, al contrario di quanto accade solitamente nel terzo turno degli insegnanti, sia frequentemente caratterizzato da feedback di contenuto e non di forma. L'assenza del turno di valutazione sulle produzioni dei partecipanti, tipica della tripletta didattica, dimostra il grado di apertura e di interesse verso lo sviluppo di una comunicazione spontanea e del dialogo critico nel gruppo. I campioni raccolti nel corso della ricerca riportano tre situazioni tipiche: i registi che commentano il contenuto del turno precedente, dimostrando comprensione e sintonia con quanto espresso dai partecipanti; i registi che si dimostrano disinteressati a correggere la forma del secondo turno e infine l'interesse ad ampliare e approfondire quanto detto 
dall'interlocutore, esattamente come potrebbe accadere in una conversazione spontanea tra pari.

Tabella 5.

\begin{tabular}{|l|l|}
\hline $\begin{array}{l}\text { Terzo turno dei registi: } \\
\text { feedback di contenuto e non di } \\
\text { forma }\end{array}$ & Esempi: \\
\hline \multirow{1}{*}{$\begin{array}{l}\text { Giorno 5 Lab1 } \\
\text { T12*IRE: tu sei molto coraggiosa (\%guardando AIK) } \\
\text { T13*FRA: le mamme che non dormono sono molto } \\
\text { coraggiose! sì, capisco bene! }\end{array}$} & $\begin{array}{l}\text { Giorno 4 Lab1 } \\
\text { T17*IND: io problema\# bisogno permesso di } \\
\text { soggiorno\# perché parlo italiano\# niente! } \\
\text { parlo inglese\# no niente! solo cinese! } \\
\text { (\%ridendo) }\end{array}$ \\
\cline { 2 - 3 } & \begin{tabular}{l} 
T18*FRA: difficile \\
\hline
\end{tabular} \\
\hline
\end{tabular}

Tuttavia, per quel che riguarda la tripletta didattica, l'elemento più significativo che abbiamo rilevato riguarda il primo turno dei registi: il vero responsabile dello sviluppo di un'interazione spontanea (Nuzzo, Grassi, 2016). La natura del primo turno del docente è, nella maggior parte dei casi, di esibizione: viene posta una domanda, di cui l'insegnante conosce già la risposta per controllare che gli studenti abbiano compreso il messaggio e per verificare di ricevere la risposta che ci si aspetta. Nel corso dei due laboratori di teatro sociale, abbiamo invece rilevato un uso costante, da parte dei registi, di domande vere o referenziali, di cui quindi non si conosce la risposta. La maggior parte di queste domande si concentra nelle attività della fase centrale del laboratorio, quella in cui i registi cercano di dare spazio alla condivisione delle storie e del vissuto dei partecipanti. Gli esempi che seguono rientrano nell'attività sul tema del coraggio, in cui i registi invitano i partecipanti a condividere con il gruppo modelli di persone o personaggi coraggiosi. I registi non possono sapere quali risposte riceveranno dai partecipanti e, anche per questo, sono realmente interessati ad ascoltare e a raccogliere i commenti dei partecipanti. Questo tipo di domanda, che quindi si avvicina notevolmente alle conversazioni spontanee, si ricollega alla questione dei quattro tipi di dominanza: i registi, non sapendo quale sarà la storia che i partecipanti condivideranno, non possono sapere in precedenza dove porterà la conversazione e quali argomenti si affronteranno. Proponendo domande vere i registri si dimostrano quindi disposti ad abbandonare anche il quarto tipo di dominanza, quella semantica, in cui nelle conversazioni asimmetriche si ha il controllo degli argomenti. 
Tabella 6.

\begin{tabular}{|l|l|}
\hline $\begin{array}{l}\text { Primo turno dei registi: } \\
\text { domande vere o referenziali e } \\
\text { rinuncia alla dominanza } \\
\text { semantica }\end{array}$ & Esempi: \\
\hline & $\begin{array}{l}\frac{\text { Giorno 4 Lab1 }}{\text { T09*FRA: ed è difficile prendere cora\# è difficile }} \\
\text { prendere coraggio! È vero no? Altre cose che } \\
\text { vogliamo dire? Coraggio? }\end{array}$ \\
\hline & $\begin{array}{l}\text { Giorno 4 Lab 1 } \\
\text { T01*IRE: dai raccontaci qualcosa, sil Un personaggio } \\
\text { coraggioso per te! (\%rivolgendosi a IND) }\end{array}$ \\
\hline
\end{tabular}

I registi sono infatti mossi dalla pedagogia del Teatro Sociale, il cui intento è di costruire uno spazio di sospensione del giudizio, sviluppando relazioni di fiducia reciproca affinché ciascuno si senta libero di condividere il proprio vissuto personale all'interno del gruppo. Attraverso la rinuncia di almeno una delle quattro dominanze che caratterizzano le conversazioni diseguali, i registi ridistribuiscono il potere fra gli interlocutori e, di conseguenza, si manifesta una maggiore apertura e disponibilità da parte dei partecipanti a parlare di sé, a condividere il proprio vissuto e a lasciarsi coinvolgere nelle attività. Tuttavia, i registi teatrali mantengono il ruolo di regia della conversazione: infatti, come si può evincere dalle tabelle sopra riportate, i registi rinunciano soltanto a un tipo di dominanza alla volta.

\section{CONCLUSIONI}

La presente ricerca ha seguito il filone di studi che indaga le pratiche didattiche attraverso l'analisi dell'interazione in classe, nella convinzione che queste analisi promuovano la consapevolezza degli insegnanti sulle proprie pratiche e sul comportamento degli studenti (Pugliese, 2020; Kunitz, 2019). Si può considerare il presente studio come una proposta di ampliamento di orizzonte delle scuole di italiano per persone migranti. In questi luoghi l'insegnante tende ad essere il protagonista principale dell'interazione scolastica, concentrandosi su una lingua di prima necessità, funzionale al soddisfacimento dei bisogni immediati.

Lo studio ha voluto dimostrare che, adottando pratiche mirate alla ridistribuzione della gerarchia interazionale, $i$ discenti possono riappropriarsi di un maggiore spazio di parola e quindi avere maggiore possibilità di output $\mathrm{e}$, di conseguenza, favorire l'apprendimento della L2. Inoltre, attraverso la pedagogia del teatro sociale si offrono situazioni comunicative reali e si permette ai discenti di ritrovare la parola nella L2 e i mezzi per esprimere la propria persona. L'insegnante, ponendosi in una condizione di ascolto attivo, rinuncia a una parte del potere interazionale e permette ai partecipanti di essere parte 
attiva nella scelta dei temi dell'interazione e nella struttura della lezione, incentivandone l'autodeterminazione.

Le precedenti (e successive) edizioni del lab.1, in cui chi scrive ha preso parte, hanno inoltre dimostrato che tali pratiche permettono a tutti i partecipanti, anche a quelli non scolarizzati o con scarso livello di competenza comunicativa, di trovare il proprio spazio di espressione. Adottando strategie come la traduzione, il ricorso al repertorio plurilingue, la cooperazione e l'uso di altri mezzi come la danza, il canto e lo sport, ogni partecipante può condividere con il gruppo l'espressione della propria persona.

Fra i differenti limiti della presente ricerca si può senz'altro evidenziare la consapevolezza del non poter paragonare un laboratorio di teatro sociale a una classe di lingua. Il primo è gestito e organizzato da registi esperti nel settore, il secondo da insegnanti, che non hanno esperienza di teatro e che si trovano a gestire le problematiche del contesto classe. Tuttavia, la convinzione di chi scrive è che non sia necessario essere un esperto di teatro sociale per poterne abbracciare la pedagogia o adottarne alcune pratiche all'interno di un percorso didattico. L'insegnante, ponendosi in una condizione di ascolto attivo, può lavorare su contenuti proposti dagli studenti e costruire lezioni di lingua che abbiano come protagonista la parola degli studenti e non solamente la lingua di prima necessità, stabilita a priori dall'insegnante o dai libri di testo.

Sarebbe certamente importante osservare le esperienze didattiche che adottano all'interno delle proprie classi di lingua tali tecniche. Sul territorio italiano troviamo scuole che abbracciano la pedagogia del teatro sociale, come Asinitas ${ }^{20}$ a Roma e Asnada ${ }^{21}$ a Milano, le cui esperienze di classe sono prima di tutto esperienze di comunità, al cui centro delle lezioni vi è l'attenzione alla persona, la libertà di espressione e la determinazione di sé (Rapetti, 2021).

\section{RIFERIMENTI BIBLIOGRAFICI}

Amoruso M., D’Agostino M., Yousif Latif Jaralla (2015), L'italiano per accompagnare. Un percorso di azione e (anche) di ricerca, in Dai barconi all'università. Percorsi di inclusione linguistica per minori stranieri non accompagnati, Scuola di Lingua italiana per Stranieri Università degli Studi di Palermo, Luxograph, Palermo, pp. 17-5.

Andorno C., Valentini A., Grassi R. (2017), Verso una nuova lingua. Capire l'acquisizione di L2, UTET, Torino.

Balboni P. (2015), Le sfide di Babele. Insegnare le lingue nelle società complesse, UTET, Torino.

Bazzanella C. (1994), Le facce del parlare. Un approccio pragmatico all'italiano parlato, La Nuova Italia Editrice, Firenze.

Bernardi C. (2004), Il teatro sociale. L'arte tra disagio e cura, Carocci, Roma.

Bernardi C., Cuminetti B. (a cura di) (1998), L'ora di teatro. Orientamenti europei ed esperienze italiane nelle istituzioni educative, Euresis, Milano.

Bernardi C., Cuminetti B., Dalla Palma S. (a cura di) (2000), I fuoriscena. Esperienze e riflessioni sulla drammaturgia nel sociale, Euresis, Milano.

Bernardi C., Dragone M., Schininà G. (a cura di) (2002), Teatri di guerra e az̧ioni di pace, Euresis, Milano.

20 https://www.asinitas.org/.

${ }^{21}$ http://www.asnada.it/. 
Caon F., Tonioli V. (2016), La sfida delle classi ad abilità linguistiche differenæiate (CAD) in Italia e in Europa, Edizioni Ca' Foscari, Venezia.

Chini M., Bosisio C. (a cura di) (2014), Fondamenti di glottodidattica. Apprendere e insegnare le lingue oggi, Carocci, Roma.

Ciliberti A. (1994), Manuale di glottodidattica. Per una cultura dell'insegnamento linguistico, La Nuova Italia, Firenze.

Cognini E. (2019), "Plurilinguismo e intercomprensione nella classe di italiano L2 a migranti adulti. Dalla lingua veicolare alla lingua-ponte", in EL.LE, 8, 1, pp. 121137.

Comodi A. (1999), "Fare teatro, fare lingua nella didattica dell'italiano L2", in Annali dell'Università per Stranieri di Perugia, nuova serie VII, 26, pp. 17-42.

D’Agostino M., Lo Maglio A. (2018), "Profili dei migranti: competenze linguistiche e alfabetizzazione", in D'Agostino M. (a cura di), La forza della lingua. Percorsi di inclusione per soggetti fragili, Scuola di lingua italiana per stranieri - Università di Palermo, pp. 23-29.

D’Agostino M., Sorce G. (2016), "CPIA Palermo 1 e Itastra: due scuole migranti”, in D'Agostino M., G. Sorce G. (a cura di), Nuovi migranti e nuova didattica: esperienze al CPLA Palermo 1, Scuola di lingua italiana per stranieri - Università di Palermo, pp. 11-22.

D’Angelo L., Di Rago R. (a cura di) (2009), Teatro, Didattica Attiva, Intercultura. Teatri visibili $e$ Teatri invisibili, FrancoAngeli, Milano.

De Meo A., Maffia M. (2021), “Oltre “no buono”. L'espressione di gusti e preferenze nella scrittura in italiano L2 di apprendenti vulnerabili", in Fiorentino G., Ceci E., Citraro C., Montinaro A. (a cura di), Alfabetizzazione come pratica di cittadinanza: teorie, modelli e didattica inclusiva, n. monografico di Lingue e Linguaggi, 41, Università del Salento, pp. 119-135:

http://siba-ese.unisalento.it/index.php/linguelinguaggi/article/view/23296.

Della Putta P. (2021), "Caratteristiche ed esiti di un corso di formazione per insegnanti volontari di italiano L2. Suggerimenti per favorire lo sviluppo di prassi didattiche efficaci", in Caruana S., Chircop K., Gauci P., Pace M. (a cura di), Politiche e pratiche per l'educazione linguistica, il multilinguismo e la comunicazione interculturale, Edizioni Ca' Foscari - Digital Publishing, Venezia, pp. 237-250:

https://edizionicafoscari.unive.it/it/edizioni4/libri/978-88-6969-502-

5/caratteristiche-ed-esiti-di-un-corso-di-formazione/.

Diadori P., Palermo M., Troncarelli D. (2015), Insegnare l'italiano come seconda lingua, Carocci, Roma.

Dragone M. (2000), "Esperienze di teatro sociale in Italia", in Bernardi C., Dragone M., Schininà G. (a cura di), Teatri di guerra e az̧ioni di pace, Euresis, Milano, pp. 45-84.

Fele G. (2003), L'interazione in classe, il Mulino, Bologna.

Fele G. (2007), L'analisi della conversazione, il Mulino, Bologna.

Fiorucci M. (2014), "Buone pratiche e criticità emerse: un bilancio e un rilancio", in Catarci M. (a cura di), L'ABC della cittadinanza. Indagine sulle Scuole di italiano per stranieri promosse dall'associazionismo a Roma e provincia, FrancoAngeli, Milano, pp. 128154.

Fonio F. (2013), "La pratica teatrale come strumento per l'apprendimento dellitaliano lingua straniera: un tentativo di bilancio e nuove prospettive", in Ribera Ruiz de Vergara A. I. (a cura di), Pratiques et enjeux de la didactique des langues anjourd'bui, Epilogos 4, Publications de l'ERIAC, Université De Rouen Normandie, pp. 21-38: http://eriac.univ-rouen.fr/wpcontent/uploads/2014/04/06PEDLAp21Fonio.pdf. 
Galli E. (2018), "Improvvisazione e glottodidattica: tecniche teatrali per l'apprendimento linguistico", in Bollettino Itals, 16, 74, pp. 23-50.

Grassi R. (2017), "Interazione e acquisizione", in Andorno C, Valentini A., Grassi R., Verso una nuova lingua. Capire l'acquisizione di L2, UTET, Torino, pp. 255-298.

Kunitz (2019), "Che cosa succede durante il lavoro di gruppo nelle classi di lingua straniera?", in LEND. Lingua e nuova didattica, 4, pp. 8-17.

MacWhinney (1995), The CHILDES project: tools for analyzing talk, Erlbaum, Hillsdale NJ.

Magnani M. (2002), "Il teatro nella glottodidattica: un'esperienza tra teoria e prassi", in Linguae \&-Rivista di Lingue e culture moderne, 1, 2, pp. 49-65.

Markee N. (2015), The Handbook of Classroom Discourse and Interaction, Wiley-Blackwell, Oxford.

Minardi S. (2020), "Le dimensioni linguistiche nel curricolo di una disciplina detta non linguistica", in Italiano LinguaDue, 12, 1, pp. 1007-1020:

https://riviste.unimi.it/index.php/promoitals/article/view/13970.

Minuz F. (2014), "La didattica dell'italiano in contesti migratori”, in Gentes, 1, pp. 107-112.

Minuz F. (2005), Italiano L2 e alfabetizzazione in età adulta, Carocci, Roma.

Nuzzo E., Grassi R. (2016), Input, Output e Interazione nella didattica delle lingue, Bonacci, Loescher, Torino.

Orletti F. (2000), La conversazione diseguale, Carocci, Roma.

Pallotti G. (2012), La seconda lingua, Bompiani, Milano.

Piazzoli E. (2009), "Metodologia Process Drama e Competenza Interculturale", in Culturiana, 3, pp. 69-73.

Pontremoli (2005), Teorie e tecniche del teatro educativo e sociale, UTET, Torino.

Pugliese R. (2020), "In italiano e in altre lingue, a casa e a scuola: le interazioni tra pari nella socializzazione linguistica", in LEND. Lingua e nuova didattica, 1, pp. 25-39:

Rapetti F. (2021), “L'alfabeto con meno parabaleni. Una proposta per un'educazione alla letto-scrittura in italiano L2 con giovani e adulti scarsamente o non alfabetizzati nella L1 e scarsamente o non scolarizza", in Fiorentino G., Ceci E., Citraro C., Montinaro A. (a cura di), Alfabetizzazione come pratica di cittadinanza: teorie, modelli e didattica inclusiva, n. monografico di Lingue e Linguaggi, 41, Università del Salento, pp. 181-197.

Rastelli S. (2010), "Il programma Marco Polo", in Rastelli S. (a cura di), Italiano di cinesi, italiano per cinesi, Guerra Edizioni, Perugia, pp. 9-22.

Sacks H. (2007), L'Analisi della Conversazione, Armando, Roma.

Sacks H., Schegloff E. A., Jefferson G. (1974), "A simplest systematics for organization of turning-taking for conversation", in Language, 50, pp. 696-735.

Salza I. (2017), "La danza delle parole. Un laboratorio teatrale per donne migranti alla scoperta della lingua italiana", in Educarione Interculturale, 15, 2, pp. 115-124: https://rivistedigitali.erickson.it/educazione-interculturale/it/visualizza/pdf/117.

Trulli M. (2014), "Profilo e pratiche delle organizzazioni coinvolte nell'indagine", in Catarci M. (a cura di), L'ABC della cittadinanza. Indagine sulle Scuole di italiano per stranieri promosse dall'associazionismo a Roma e provincia, FrancoAngeli, Milano, pp. 2768.

Tsui A. (1996), "Reticence and anxiety in second language learnig", in Bailey K., Nunan D. (eds.), Voices from the language classroom, Cambridge University Press, Cambridge, pp. 151-153.

Vedovelli M., Villarini A. (2003), "Dalla linguistica acquisizionale alla didattica acquisizionale: le sequenze didattiche nei materiali di italiano L2 destinati agli immigrati stranieri", in Giacalone Ramat A. (a cura di), Verso l'italiano, Carocci, Roma, pp. 270-304. 